
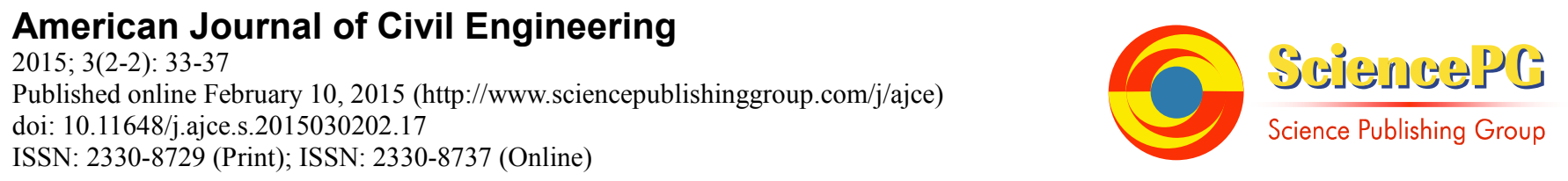

\title{
Evaluating methods for 3D CFD Models in sediment transport computations
}

\author{
Hamid Reza Madihi*, Sina Bani Amerian \\ Graduate Faculty of Environment, Tehran University, Tehran, Iran \\ Email address: \\ hrmadihi@ut.ac.ir (H.R. Madihi), Sina_Bani Amerian@ut.ac.ir (S. Amerian)
}

\section{To cite this article:}

Hamid Reza Madihi, Sina Bani Amerian. Evaluating Methods for 3D CFD Models in Sediment Transport Computations. American Journal of Civil Engineering. Special Issue: Research and Practices of Civil Engineering in Developing Countries. Vol. 3, No. 2-2, 2015 , pp. 33-37. doi: 10.11648/j.ajce.s.2015030202.17

\begin{abstract}
Usual uncertainties in computational fluid dynamics (CFD) results include numerical errors, modeling errors, program bugs, mistakes in input parameters and boundary conditions. The errors can be assessed using itemized results from the CFD program together with its documentation. Each uncertainty can be assessed by evaluating the variables against each other, parameter responsiveness examinations and testing for simplified items. The role is made easier if the program is as transparent as possible. This means that the user can see the values of all the pertinent variables and mediatory results for the whole computational domain. Together with an extensive collection of documents of the computer program that includes all the formulas used, the user can be able to find the causes of suspect results, including an estimation of possible bugs. An important tool in the testing of a CFD program is using simplified cases, generally channels with uniform one-dimensional flow.
\end{abstract}

Keywords: Sediment, Models, CFD, Transport

\section{Introduction}

Engineering investigations of sediment transport problems have until recent years been done thorough physical model studies. Laboratory modeling have the disadvantage of several scaling problems, for example modeling the interaction between bed load and suspended load, and modeling bed forms. The scaling problems have often necessitated very large laboratory models, which are costly and time-consuming to build. An alternative approach that has evolved with the increased speed of computers in recent years is to calculate bed elevation changes by using of computational fluid dynamics (CFD).

The Navier-Stokes equations are solved in three dimensions together with equations for sediment transport. Empirical formulas are used for critical bed shear stress, roughness estimations, bed form effects etc. The approach complicated and it takes several years to make a computer program capable of carrying out the task. The number of people who have made such programs is therefore limited, and most of engineers involved in sediment transport studies will only use programs others have made. It is technically possible to use the programs without knowing the details of the numerical algorithms. Generating the input data is not always too complicated, and the models will then produce a result, also for an inexperienced user. The main problem is to assess how accurate and reliable the results will be. Some method is needed to address this problem and ensure sufficient quality of the results. Knowledge about numerical modeling theory is essential in this solution. Establishments of best practice guidelines would be very useful.

\section{Data and Material}

It is often not too complicated to produce results using commercial CFD programs. The main problem is to find out how accurate the results are, and if they are good enough for practical engineering purposes. An organization that has worked on this topic is ERCOFTAC. ERCOFTAC is an acronym for European Research Community on Flow, Turbulence And Combustion [1]. The organization has produced a set of best practice guidelines for CFD. Included is a list of errors and uncertainties in CFD. The list includes the following topics:

- Errors in numerical approximations

- Modeling errors

- Errors in input data

- Programming errors 
Best practice would involve an assessment of these errors and uncertainties. Errors in the numerical approximations include false diffusion, arising from a grid that is too coarse and/or inaccuracies in the discretisation schemes. The false diffusion can be investigated by varying the grid size or using different discretization methods. The difference between the results with the varying parameters is an estimation of the errors due to false diffusion. A CFD program should make it easy for the user to vary the grid size and make parameter tests with different grid resolutions. The program should also include multiple discretization schemes with varying accuracy. Modeling bed elevation changes over time involves a time-dependent computation. The total time is each time step. Because iterative solvers are used and computational time may be long, sometimes the iterations are not done until complete convergence for each time step. Then an error will be introduced $[1,2]$

Assessment of this error can be done by looking at the residual values over time or the average residual for each time step. The CFD program should therefore produce such information to the user. For most practical engineering cases, the needed input data for the CFD model is not available. Typical data needed for the model is a digital terrain model and spatial variation in bed grain size distribution at the bed. Also, a time series of inflow of sediments together with its grain size distribution can be difficult to find. To assess the errors involved in possible uncertainties in the input data, a parameter sensitivity test can be used. Errors in the computer program are also possible.

Abbreviations such as IEEE, SI, MKS, CGS, sc, dc, and a 3D CFD program with sediment transport may have over 100 000 lines of code, and bugs are therefore likely. Bugs are often difficult to find, but they may show up during a detailed analysis of the computed variables or in a thorough parameter sensitivity test. For example, it is possible to see if the empirical formulas for sediment parameters are coded correctly by viewing all the variables in a cell and computing the results by hand.

The CFD program should therefore provide the user with parameters like the water depth, bed shear stress, grain size distribution etc. Bugs may also show up by giving unphysical results for a parameter. Examples are: erosion at a section with non-erodible bed, negative sediment concentrations, high fractions of fine sediments at the bed in areas of erosion, sediment deposition at the outside of a bend instead of at the inside etc. Possible bugs can be investigated using simplified cases, as described in more detail later.

Modeling errors include the turbulence model, but also all the empirical functions related to sediment transport and bed roughness. The best way of assessing the error is to look at the results with varying parameters in the formulas. This is a standard parameter sensitivity test. There are a number of empirical parameters in the formulas, giving a number of output variables to assess.

Most CFD computations today are done in mechanical engineering, use only one fluid, smooth walls and a fixed geometry. In such a situation, the main processes causing inaccurate results are false diffusion if the grid is too coarse and inaccuracies in the turbulence model. Research on CFD in mechanical and aerospace engineering therefore have focused considerably on the turbulent processes. In using CFD for sediment transport modeling, the main uncertainty is the physics of the sediment processes. The large errors involved in the sediment computations often make small inaccuracies in the turbulence model insignificant.

\section{Research Methodology}

\subsection{Assessing Sediment Transport Results from CFD Models}

The goal of a sediment transport computation is often to predict bed changes in a fluvial system. The changes are mostly in the vertical direction, but also lateral erosion can be computed.

The basis of a vertical bed elevation change in a computational cell is the continuity of sediments flowing in and out of the cell. The sediment transport flux is a function of an empirical formula for sediment transport capacity or sediment concentration. This formula is again a function of the particle size on the bed, the bed grain size distribution and the effective bed shear stress. The bed shear stress is a function of the water velocity close to the bed, the water velocity gradient, the turbulent kinetic energy, the bed form sizes and the roughness. The roughness and the bed form height is a function of the grain size distribution, the water depth and the sediment fluxes $[1,2,10]$.

The water velocity and the turbulence is a function of the roughness, the geometry of the case, the water level, discharge, the grid and the turbulence model. Another process taking part is secondary currents. Experienced and knowledgeable scientists may also use a CFD program to compute sediment transport and not get the results they see in the field or in the laboratory. If the program is a black box, it is impossible for the scientist to know why the results are incorrect. The present paper proposes features of the program, which allows for transparency of the algorithms used with intermediate results. The user is thereby given the possibility to follow the computations and see where the problem arises. Together with information about the algorithms and empirical formulas used by the program, the user can assess the need to make improvements in the program.

Most CFD programs will provide an output of the computed water velocity field and turbulence parameters. A program computing bed elevation changes must of course also show this variable graphically. A transparent CFD program will also show all other variables that are used in the computation of the bed elevation. In relation to computing the water velocity, the following variables are useful:

- Bed shear stress

- Roughness

- Water depth

- Froude number

The bed shear stress is the most important parameter for the 
sediment transport computations, so this parameter needs to be shown graphically. Comparing the water velocity field close to the bed with the bed shear stress should show a similar pattern. The shear stress is also a function of the bed roughness, so it is important to both parameters into account in the assessment. The water depth is also an important parameter. Using a structured grid, the aspect ratio of the cells will be unfavorable in shallow areas and can lead to convergence problems. For unstructured grids with a varying number of cells in the vertical direction, this will not be a problem. The number of cells in the vertical direction can also be shown, to check if the values correspond well to the water depths, and that there are not too many cells in shallow areas. The Froude number is important for free surface algorithms. Areas of Froude number above unity indicate possible existence of hydraulic jumps, which often cause problems for these algorithms. Instabilities may be identified by looking at the water continuity [13]. Using the SIMPLE method, the residual for the pressure-correction equation is the water continuity defect for each time step [10]. Looking at this variable in a graphics plot can identify problematic areas of instabilities. Watching the velocity vectors as they, change over time can also give useful information.

For sediment computations, important parameters are:

- Sediment concentration for all size fractions

- Bed grain size distribution

- Bed form height

- Sediment transport capacity

- Thickness of sediment layer

The sediment transport concentration close to the bed should be strongly related to the bed shear stress. It is possible to use hand computations with the empirical formulas to control if the computer program computes the correct values. The bed elevation changes are again related to the sediment concentration in the cells near the bed. A high sediment concentration at the bed will lead to erosion, and a decreasing concentration gradient along the direction of the bed velocity vectors will lead to deposition. If the bed shear stress is high and the concentration is not, then the reason can be that the thickness of the available sediment layer may not be sufficiently high. This can then be checked by looking at this parameter. Using a non-uniform sediment grain size distribution in a CFD program is most often done by modeling multiple sediment sizes. Then sediment concentrations for each size could be checked. If there is low concentration of one size, this may be caused by the limited availability of this particular size in the bed material in the particular cell. This can be checked by looking at graphics of the grain size distribution. The bed form height affects the sediment transport capacity by increasing the roughness and thereby the bed shears stress. Note the shear stress partition between the friction due to the sediment grains and the bed forms will also affect the effective bed shear stress used in the computation of the sediment concentration.

The sediment transport will be affected by the secondary currents that exist in bends and around obstacles. The secondary current can be shown graphically by displaying both the velocity vectors at the bed and at the water surface at the same time (Fig. 2). The angle between the vectors will be a function of the strength of the secondary current. This angle may also be displayed as a variable in a contour map (Fig. 1). The figure with the bed and surface vectors can also be used to check that the computed velocity field is in the correct direction in bends.

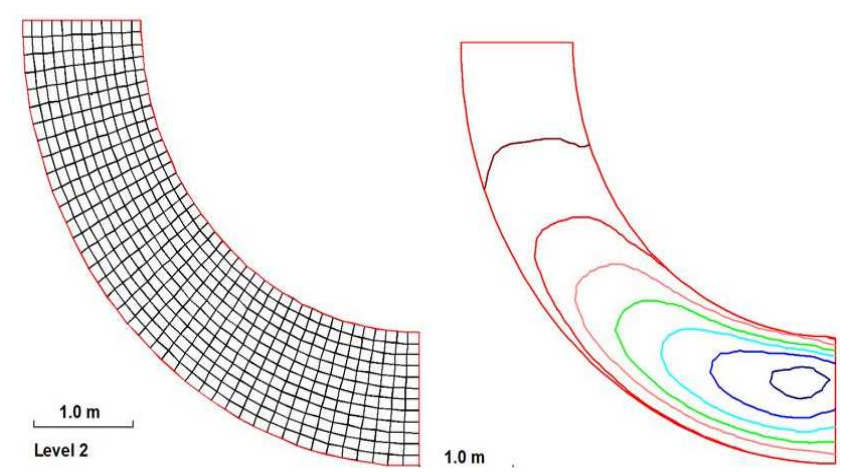

Figure 1. Plan view of the grid (left) for the bend. The water is flowing in from above and out to the right.The right figure shows a contour map of the secondary current angle. The dark blue value is -14 degrees. The dark red value is - 2 degrees.

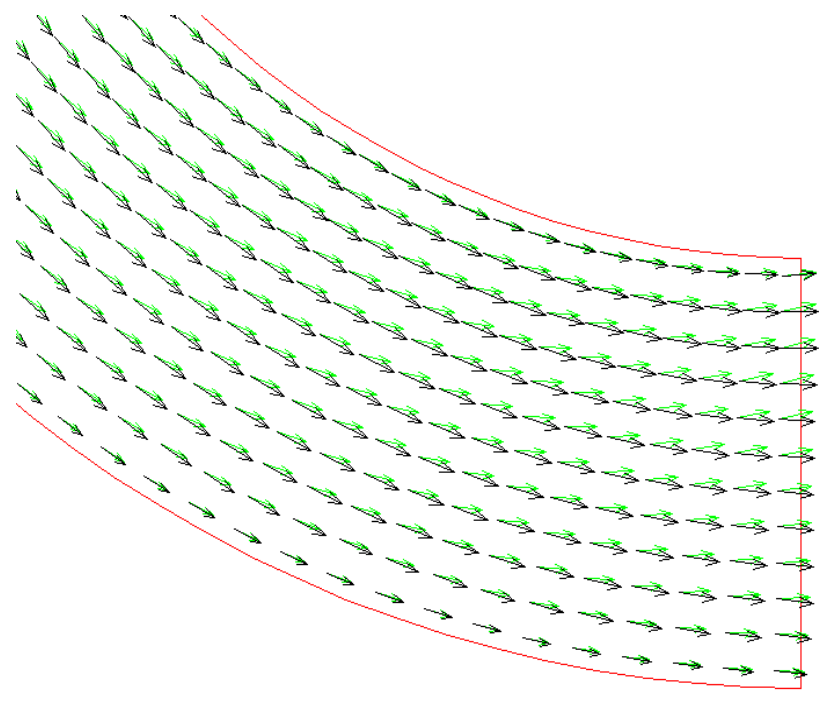

$0.8 \mathrm{~m}$

Figure 2. Velocity vectors at the surface and the bed for flow in a bend, showing the secondary current

\subsection{Testing for Simplified Situations}

The case should be designed so that focus is given on one effect, and other processes do not affect the results. An example is if the user wants to find out if the program computes the correct bed shear stress. A straight flume can be modeled with zero gradient boundary conditions on the sides, ensuring a 1D situation. The resulting pressure gradient can then be compared with hand calculations using for example Manning's formula. The same approach can be used to compute the vertical velocity profile and compare it with the logarithmic formula of Schlichting [4]. Similarly, the vertical profiles of turbulence parameters or sediment concentrations can be tested. The 1D case can also be used to test other 
parameters against empirical formulas, for example the sediment transport capacity, bed form height etc. Simplified geometries are also used for test cases when validating computer programs and numerical algorithms.

Examples are flow over a backward facing step or cavity flow to test the turbulence model. The length of the recirculation zone is then the main testing parameter. A collapsing water column or a wave can be used for testing free surface algorithms Simplified cases do not always have to involve a simplified geometry [3].

A CFD program computing sediment transport will also include algorithms for turbulence, free surface location, roughness effects etc. It is possible to test the accuracy of these algorithms without computing the sediment transport. An example is given in Fig: 1, where the flow in a bend is computed. The water surface algorithm will then give a transverse slope of the channel. This can be compared with simple analytical formulas for the centrifugal acceleration related to gravity.

\section{Result and Analysis}

Some CFD programs use a primitive turbulence model that contains calibration parameters. An example is the use of a constant-eddy viscosity model for the horizontal directions in some programs solving the shallow-water equations. However, most general-purpose CFD programs will use an advanced turbulence model that does not need calibration, for example the k-epsilon model.

The main calibration parameter for solving the Navier-Stokes equations will then be the bed roughness. This calibration parameter is physical, and is also used in laboratory model studies. Modeling bed elevation changes in rivers, it is possible to use empirical formulas that relate the sediment grain size distribution and the bed form height to the bed roughness. If these formulas were exactly correct, the CFD model would in principle not need any calibration. However, most of the empirical functions describing the sediment flow have large uncertainties and low accuracy. This applies for example for the formulas for critical bed shear stress for multiple grain size mixtures, effect of cohesive forces, bed form height, roughness as a function of grain size distribution and bed form height, and the formula for the sediment transport capacity itself. It is a well accepted fact that

The sediment transport capacity formula will have less accuracy than $30-40 \%$ for many cases $[8,9,11]$. The large number of formulas giving highly different predictions is an evidence for this. When empirical formulas with so large inaccuracies are used in a CFD model, the results will also have similarly large errors, even if the velocity field and turbulence would be theoretically exactly computed $[5,6,7]$. Then calibration of the empirical parameters may be the only option of ensuring some reliability in the CFD results.

In the future, we can hope that more accurate empirical formulas will be developed. Then it would be possible to compute bed elevation changes in rivers without calibration. This would be a great advantage for cases assessing the impact of future man-made impacts in rivers, where calibration data do not exist.

\section{Discussion and Conclusions}

Three-dimensional numerical models for sediment transport should be designed as transparent as possible, providing the user with graphical output of all relevant parameters. Also, a thorough documentation of all equations and empirical formulas used by the program should be given, enabling the user to assess the computational procedures of the program to see possible bugs.

When testing numerical model results against observed bed elevation changes, a procedure should be used where the user look at the values from all the parameters and checks the empirical formulas used in the program. Starting from the velocity field to the bed elevations changes, the parameter distribution in the geometry should be investigated to see if it is reasonable. How each variable changes over time can also give very useful information.

When using 3D CFD model to predict bed elevation changes for a situation where no calibration data exist, best practice should be to investigate all uncertain parameters and formulas in parameter sensitivity tests. A large number of parameters should be tested, from grid size, discretization schemes, turbulence models, values for critical bed shear stress parameters, empirical coefficients in the sediment transport capacity formula, parameters in bed form and roughness prediction formulas etc. The test will give the total accuracy of the results and expose influence of each parameter on the final result.

If the user is in doubt whether the CFD program computes the correct result, a testing should be done for a simplified case, focusing on the potential problem. A typical simplification is to model a straight flume with uniform, steady flow using a very coarse grid. Such a case can expose errors in the computation of the shear stress on the bed or the sediment transport formula. If the problem is believed to be in the free surface or water flow part of the program, then the simplified case could be run without sediments.

The main uncertainty in today's prediction of bed elevation changes using 3D CFD models is the empirical formulas for the sediment transport processes. Future research should therefore focus on improving the accuracy of these formulas. This is an area where physical model studies will be needed for many years to come.

\section{References}

[1] Baranya, S. and Jozsa, J.) "Morphological modeling of a sand-bed reach in the Hungarian Danube", Proceedings of the 33rd Congress of the International Association of Hydraulic Engineering and Research, Vancouver, Canada, 2009.

[2] T. Fischer-Antze, N. R. B. Olsen. and D.Gutknecht, "Three-dimensional CFD modeling of morphological bed changes in the Danube River", Water Resources Research, 44, W09422,doi:10.1029/2007WR006402,2008. 
[3] T.Fischer-Antze,N., Ruether,.,N.R.B Olsen, and D .Gutknecht,. "3D modeling of non-uniform sediment transport in a channel bend with unsteady flow", Journal of Hydraulic Engineering and Research, Vol. 47, No. 5, pp. 670-675, 2009.

[4] H. Schlichting, "Boundary layer theory", McGraw-Hill, 1979.

[5] M.Abboltt, An introduction to the Method of characteristics, Thames and Hudsun, 1966.

[6] M.Abotte, Computational Hydraulics, Pitman, 1979.

[7] F.Henderson,Open Channel Flow, Macmillan,1996.

[8] W.Gray and G.. Pinder, On the relationship between the FEand FD method, Int.j.Num. Methods Engng, Vol.12, No.9, 1976.
[9] S.Nakamura, Computational Method Engineering and Science, Wiley,1997.

[10] A.Raudkivi and R Callander, Advanced Fluid Mechanics, Arnold, 1975.

[11] R.H Gallagher(ED).Finite Element Techniques for Fluid Flow, Newnes Bruteerworths, 1976.

[12] R. Hamming, Introduction to Applied Numerical Analysis, McGraw-Hill,1971.

[13] E.Isaaacson and H. Keller, Analysis of Numerical Methods, Willy, 1966. 